\title{
BMJ Open Virtual reality intervention to improve apathy in residential aged care: protocol for a multisite non-randomised controlled trial
}

\author{
Dimitrios Saredakis (D , ${ }^{1}$ Hannah A D Keage, ${ }^{1}$ Megan Corlis, ${ }^{2}$ Tobias Loetscher $^{1}$
}

To cite: Saredakis $D$, Keage HAD, Corlis M, et al. Virtual reality intervention to improve apathy in residential aged care: protocol for a multisite non-randomised controlled trial. BMJ Open 2021;11:e046030. doi:10.1136/ bmjopen-2020-046030

- Prepublication history and supplemental material for this paper are available online. To view these files, please visit the journal online (http://dx.doi org/10.1136/bmjopen-2020046030).

Received 19 0ctober 2020 Revised 19 December 2020 Accepted 15 January 2021

Check for updates

(c) Author(s) (or their employer(s)) 2021. Re-use permitted under CC BY-NC. No commercial re-use. See rights and permissions. Published by BMJ.

${ }^{1}$ UniSA Justice \& Society, University of South Australia, Adelaide, South Australia, Australia

${ }^{2}$ UniSA Clinical \& Health Sciences, University of South Australia, Adelaide, South Australia, Australia

Correspondence to Mr Dimitrios Saredakis; dimitrios.saredakis@mymail. unisa.edu.au

\section{ABSTRACT}

Introduction Apathy is a prevalent neuropsychiatric symptom for older adults residing in aged care. Left untreated, apathy has been associated with accelerated cognitive decline and increased risk of mortality. Reminiscence therapy is commonly used in aged care and has demonstrated to reduce apathy. Traditional methods of reminiscence use physical objects and more recently technology including tablets and laptop computers have demonstrated potential. Virtual reality (VR) has successfully been used to treat psychological disorders; however, there is little evidence on using VR for behavioural symptoms such as apathy in older adults. Using VR to deliver reminiscence therapy provides an immersive experience, and readily available applications provide access to a large range of content allowing easier delivery of therapy over traditional forms of therapy. This study aims to identify changes in apathy after a reminiscence therapy intervention using head-mounted displays (HMDs).

Methods and analysis Participants will be allocated to one of three groups; reminiscence therapy using VR; an active control using a laptop computer or physical items and a passive control. A total of 45 participants will be recruited from residential aged care (15 in each group). The three groups will be compared at baseline and follow-up. The primary outcome is apathy, and secondary outcomes include cognition and depression. Side effects from using HMDs will also be examined in the VR group. Primary and secondary outcomes at baseline and followup will be analysed using linear mixed modelling.

Ethics and dissemination Ethics approval was obtained from the University of South Australia Human Research Ethics Committee. The results from this study will be disseminated through manuscript publications and national/international conferences.

Trial registration number ACTRN12619001510134.

\section{INTRODUCTION}

The presence of apathy can be disabling for residents in long-term aged care facilities. ${ }^{1}$ Having apathy results in diminished goal-directed behaviour. ${ }^{2}$ Therefore, individuals with apathy are more likely to withdraw from care, ${ }^{3}$ therapeutic activities ${ }^{4}$ and have reduced self-care behaviours. ${ }^{5}$ In aged care facilities, neuropsychiatric symptoms occur

\section{Strengths and limitations of this study}

This study is the first to compare the use of virtual reality (VR) for reminiscence with traditional methods and a usual care group.

- The results of this study will provide evidence for using VR to reduce levels of apathy for older adults in residential aged care.

- Sampling method may introduce selection bias.

- Sample will most likely include participants with different neurological conditions making generalisability difficult.

in approximately $90 \%$ of people, ${ }^{6}$ and the prevalence of apathy has been reported to vary between $44 \%$ and $84 \%{ }^{7-10}$ It is common for apathy to go undiagnosed or less likely to be treated. ${ }^{11}{ }^{12}$ However, patients with apathy can have almost a twofold increased risk of dementia. $^{3}$

Despite the prevalence and consequences of having apathy, pharmacological treatment is limited and can be difficult due to differing factors including apathy subtypes, stage of neurodegeneration and age that can influence efficacy. ${ }^{13}{ }^{14}$ Non-pharmacological treatments are a safe alternative, and there is a broad consensus that non-pharmacological approaches for treating apathy, in particular, personalised therapy using information and communication technologies should be used. $^{15}$

Reminiscence therapy is an approach used in aged care that can be individualised and has demonstrated potential. ${ }^{16}$ One of the main strengths of reminiscence therapy is that it is a person-centred approach that emphasises the importance of personal identity ${ }^{17}$ Loss of independence caused by moving into an aged care facility can cause a person's identity to deteriorate and may increase apathy. ${ }^{18}$ A meta-analysis examining the use of reminiscence for improving psychological 
well-being in older adults found an overall medium effect size of $0.54 .{ }^{19} \mathrm{~A}$ more recent systematic review and metaanalysis examining reminiscence therapy for people with dementia included 22 studies of which two studies examined apathy ${ }^{16}$ One study was included in the meta-analysis and found no difference at follow-up in apathy using the Apathy Inventory Scores after 3-month follow-up, mean difference $1.4095 \%$ CI $(-1.30$ to 4.10$),{ }^{20}$ participants were classified as having Alzheimer disease and were non-institutionalised. The second study included in the review but not the meta-analysis found improvement after 3-month follow-up using the Apathy Evaluation Scale (AES) $(\mathrm{Z}=-3.10, \mathrm{p}=0.002)$, in this study participants were institutionalised with mild-to-moderate dementia. ${ }^{21}$ Other benefits from reminiscence therapy found in this review were improvements in mood, cognition and communication, although results were inconsistent. ${ }^{16}$ This demonstrates that reminiscence therapy can work but calls for consistency in methods and alternative forms of delivery to assist with improving outcomes. Technology is now commonly used with reminiscence therapy in the form of touchscreen tablets or laptop computers, providing access to a multitude of content. ${ }^{22}$ The use of technology allows for personalisation and provides the ability to reduce the amount of time required to prepare for sessions. Videos can provide sound and audio, increasing the realism of the reminiscence experience..$^{23}$ Using immersive technologies can be a way of providing personalised therapy to improve outcomes.

Virtual reality (VR) using head-mounted displays (HMDs) is a fully immersive technology that has been successfully used to treat conditions including posttraumatic stress disorder, anxiety and pain during medical procedures. ${ }^{24-26}$ Research using VR to treat apathy in older adults is limited. A single-session study measuring apathy using HMDs found improvements in apathy $(\mathrm{Z}=-2.818, \mathrm{p}=0.005)$, this study included both group and individual sessions, used a generic library of videos and participants were limited to those with no or minimal cognitive impairment. ${ }^{27}$ Other studies using VR and looking at apathy have also been single-session studies, therefore, not examining changes in apathy. ${ }^{28} 29$ A recent feasibility study, ${ }^{30}$ found that participants with varying levels of cognitive decline ranging from minimalto-moderate impairment could tolerate the use of VR with HMDs in agreeance with previous research. ${ }^{31}$ The VR reminiscence experience in this study was specifically tailored for each participant. Improvements in verbal fluency were found after the intervention in participants with higher levels of apathy at baseline $(\mathrm{r}=0.719,95 \% \mathrm{CI}$ 0.327 to $0.900, \mathrm{p}=0.003$ ), and $35 \%$ of participants in the study did experience temporary and minor side effects from using VR.

There is increasing evidence of acceptability of using HMDs in older adults. However, users of VR can experience symptoms of motion sickness. ${ }^{32} \mathrm{~A}$ recent systematic review and meta-analysis has highlighted the lack of research in examining side effects in older adults from using VR, and although older adults reported lower symptomatology than younger samples, these findings were based on a small number of studies and excluded clinical samples. ${ }^{33}$ Additionally, research with older adults does not always assess side effects from using VR. ${ }^{34}$ There is a need to increase our understanding of side effects from using VR in older adults, particularly with the increased use of HMDs for therapy in older clinical populations.

This study will deliver reminiscence therapy to older adults aged $\geq 65$ years living in residential aged care to reduce apathy. Reducing apathy may assist with delaying the rate of cognitive decline and attenuate the expected functional decline that occurs in long-term care $^{35}$ in maintaining a person's overall health and well-being. The benefits of therapy are not limited to individuals as it may also provide more engaged residents for residential aged care facilities. Three groups will be compared: reminiscence therapy using VR (intervention group); reminiscence using a laptop computer or physical items (active control group) and usual care (passive control group). The primary hypothesis is that the VR group will have lower apathy scores than the traditional reminiscence group after the intervention. The intervention and active control groups will also be compared against the passive control group to establish the effectiveness of reminiscence therapy overall. The secondary outcomes include cognition and depression. For the VR group only, side effects from using HMDs will also be examined. During therapy for both the VR intervention and active control groups, heart rate variability, galvanic skin response and speech will be examined as exploratory outcomes, additional exploratory outcomes for all three groups include loneliness, quality of life and physical activity.

\section{METHODS AND ANALYSIS \\ Design}

This is a multisite non-randomised controlled trial. The Standard Protocol Items: Recommendations for Interventional Trials guidelines ${ }^{36}$ were used to set out the structure of this protocol. The study will include 45 adults aged $\geq 65$ years, living in residential aged care. Assessments will be performed at baseline and follow-up approximately 2 weeks apart for all three groups. Activity monitors are fitted prior to baseline and on completion of follow-up. Therefore, all participants are in the trial for approximately 3 weeks. The VR and active control groups will receive a reminiscence intervention, and the passive control will receive usual care during the trial period.

\section{Patient and public involvement}

There was no patient and public involvement in the study design.

\section{Setting}

Data collection will be conducted across three residential aged care facilities operated by an aged care provider in South Australia. Selected sites are in the northeast or 


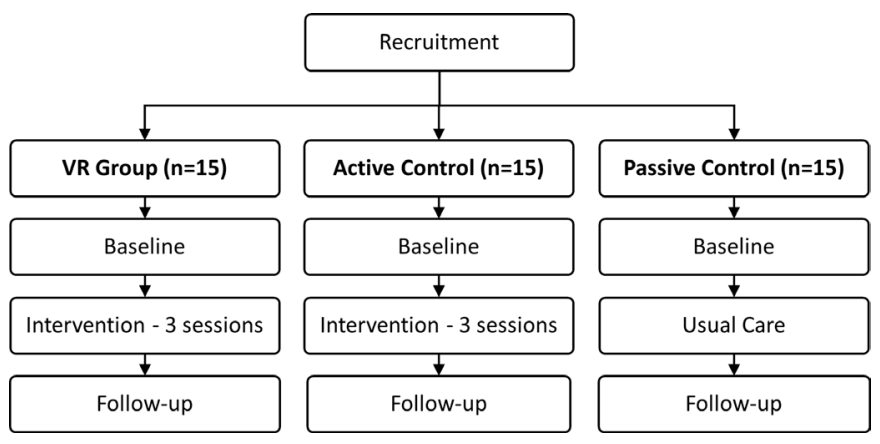

Figure 1 Participant allocation and flow chart of study procedure. VR, virtual reality.

inner northern suburbs within the metropolitan area of Adelaide, South Australia. Site allocation was not randomised and determined by the aged care provider. All three facilities provide personal and healthcare services including dementia support, respite and palliative care.

\section{Eligibility criteria}

For all three groups, participants are eligible if they are aged 65 years or older, men or women and proficient English speakers. Participants also need to be willing to undertake follow-up assessments. For the VR group, participants need to be able to tolerate wearing the HMD and have vision that can be corrected with the use of their current glasses, their glass frame also need to be able to fit into the HMD.

For all three groups, participants are excluded if: they have known learning disabilities, their score on the Psychogeriatric Assessment Scale ${ }^{37}$ as assessed by the aged care facility is 16 or higher indicating severe cognitive impairment, have significant neurological disorders and other conditions including agitation and aggression at a level that would make assessment difficult. Finally, those who have issues with confusion/disorientation or who may become distressed due to confusion re time and place.

\section{Intervention groups}

The study consists of three groups, see figure 1 . The VR group undertakes reminiscence therapy using VR. The active control undertakes reminiscence therapy using a laptop computer and/or physical items including books, magazine or photos, and the passive control receives their usual daily care.

As participants are blinded to the other groups, each group is allocated to a separate residential aged care site. Many participants socialise at mealtimes or with group activities and this will minimise awareness of other groups. With the VR group it is first established if participants can see to a satisfactory level in the HMD prior to the consent process. This is done by displaying an image in the HMD that the participant is familiar with and asking the participant to assess the clarity of the image. If the quality is acceptable and it is established that the participant can tolerate the use of the HMD, then the consent process is undertaken.

\section{Study stages}

The study is divided into three stages, baseline, intervention and follow-up.

\section{Baseline}

Baseline measures take approximately 1 hour and include demographics, apathy, depression, quality of life, a loneliness measure and a cognitive assessment, see table 1. For the VR and the active control groups, a further 1 hour (approximately) is required. This is to conduct an interview about the participant's history according to reminiscence therapy guideline ${ }^{38}$ for establishing content to be used in the intervention sessions.

Table 1 Timeline with measures and assessments used throughout the study

\begin{tabular}{|c|c|c|c|c|c|}
\hline \multirow[b]{2}{*}{ Assessments } & \multirow[b]{2}{*}{ Baseline } & \multicolumn{3}{|c|}{ Intervention } & \multirow[b]{2}{*}{ Follow-up } \\
\hline & & Session 1 & Session 2 & Session 3 & \\
\hline Activity & $x$ & & & & $x$ \\
\hline Apathy Evaluation Scale & $x$ & & & & $\mathrm{x}$ \\
\hline Addenbrooke's Cognitive Examination III & $x$ & & & & $x$ \\
\hline Quality of life & $\mathrm{X}$ & & & & $x$ \\
\hline Loneliness & $x$ & & & & $\mathrm{x}$ \\
\hline Geriatric Depression Scale & $x$ & & & & $\mathrm{x}$ \\
\hline \multirow[t]{2}{*}{ Simulator Sickness Questionnaire } & & $x$ & & $x$ & \\
\hline & & VR only & & VR only & \\
\hline Heart rate and galvanic skin response & & $x$ & & $\mathrm{x}$ & \\
\hline Speech tasks & & $x$ & & $x$ & \\
\hline Session record & & $x$ & $x$ & $\mathrm{X}$ & \\
\hline Staff questionnaire & & & & & $x$ \\
\hline
\end{tabular}

VR, virtual reality. 
During the reminiscence sessions for both intervention groups there is continual conversation, and feedback is requested after the first session to guide the content for the following sessions. The therapy may need to be flexibly tailored depending on the individual's unique life experience and reaction to the therapy. For example, some participants may have increased positive reactions to music or memories at specific stages of their life. The use of objective measures has been recommended for assessing apathy in clinical trials including actigraphy. ${ }^{39}$ Galvanic skin response, heart rate ${ }^{40}$ and more recently, speech analysis ${ }^{41}$ and their association with apathy have also been examined. Therefore, to measure rest and physical activity, all three groups have the GENEActiv wrist-worn accelerometer (Activinsights, Kimbolton, Cambridgeshire, UK) fitted for a period of 48 hours at baseline, this is repeated after follow-up. The intervention will examine other objective measures of apathy as exploratory outcomes including speech, galvanic skin response and heart rate variability.

\section{Intervention}

The intervention consists of three sessions spaced at a minimum of 1 day apart within a 2-week period. All intervention sessions are carried out individually. Both the VR and active control groups have the Empatica E4 (Empatica, Boston, USA) fitted for the duration of the session to measure heart rate variability and galvanic skin response. Event markers on the Empatica E4 are set for each task during the session to distinguish between five separate periods. These include:

1. Pre reminiscence-Speech task 1: Voice recording of the participant's response to a question (1 min).

2. Pre reminiscence-Speech task 2: Voice recording of the participant reading from a wordless picture book (up to a maximum of $5 \mathrm{~min}$ ).

3. The reminiscence component (20 min).

4. Post reminiscence-Speech task 3: Voice recording of the participant's response to a question (1 min).

5. Post reminiscence-Speech task 4: Voice recording of the participant reading from a wordless picture book (up to a maximum of $5 \mathrm{~min}$ ).

In the VR group, if a participant experiences symptoms of VR sickness that creates discomfort, the session is immediately stopped. If symptoms subside, the intervention continues; if after a second attempt symptoms continue, the participant will be requested to withdraw from the study. All content is to be carefully selected avoiding fast moving scenes, using high-quality footage with smooth motion and stabilised video. Participants are to remain seated during the VR session as this will assist to avoid VR sickness and reduce risk of falls. In the VR group, motion sickness symptoms are measured before and after the reminiscence experience. The passive control group does not undertake any intervention and receives their usual care between baseline and follow-up.

\section{Follow-up}

The follow-up session is performed the day after the final intervention session and all baseline measures are repeated. Participants in all three groups are not prevented from participating in their normal lifestyle activities during the research.

\section{Primary outcome}

\section{AES clinician version}

The presence of apathy will be assessed using the AES clinician version. ${ }^{42}$ Trained researchers rate each item based on both verbal and non-verbal responses from the participant. The AES contains 18 items measured on a scale ranging from 'not at all' to 'slightly', 'somewhat' and 'a lot'. Scores range from 18 to 72, with higher scores indicating a higher level of apathy. The clinician version of the AES has found to have a test-retest reliability of 0.88 and good internal consistency $(\alpha=0.90) .{ }^{42}$

\section{Secondary outcomes}

\section{Addenbrooke's Cognitive Examination III (ACE-III)}

Cognition will be assessed using the ACE-III, this test is a widely used cognitive screening tool that assesses attention, fluency/language, verbal memory and visuospatial function. ${ }^{43}$ The ACE-III is scored out of 100 with higher scores indicating higher levels of cognition. There are three different versions of the ACE-III for use in repeated measures testing, two versions are used in this study. The ACE-III has reported good internal consistency $(\alpha=0.88){ }^{44}$

\section{Geriatric Depression Scale (GDS) Short Form}

The GDS is a tool for detecting a person's level of depression specifically for older populations. ${ }^{45}$ This scale has a $92 \%$ sensitivity and $89 \%$ specificity when compared with diagnostic criteria ${ }^{45}$ and good internal consistency $(\alpha=0.80) .{ }^{46}$ The GDS consists of 15 items and is scored out of 15. Higher scores indicate likelihood of depression with a score equal to or greater than 10 suggesting high risk of depression. ${ }^{45}$

\section{Exploratory outcomes}

The Quality of Life in Alzheimer's Disease (QOL-AD)

The QOL-AD Scale will assess a participant's current level of quality of life. ${ }^{47}$ Originally developed for people with Alzheimer's disease, the QOL-AD has also been used for those without dementia and in residential aged care settings. The QOL-AD is a self-report measure consisting of 13 items rated on a 4-point scale. Good internal consistency has been reported $(\alpha=0.82)$ for the QOL-AD. ${ }^{48}$

\section{Three-Item Loneliness Scale}

The level of loneliness will be assessed using the ThreeItem Loneliness Scale. This scale is a shortened version of the Revised UCLA Loneliness Scale. ${ }^{49}$ The scale comprises three questions and is rated on a 3-point scale. Scores range from 3 to 9 with higher scores indicating increased levels of loneliness. The Three-Item Loneliness Scale has reported acceptable internal consistency $(\alpha=0.72){ }^{49}$ 
Simulator Sickness Questionnaire (SSQ)

Side effects from using VR will be measured using the $\mathrm{SSQ}^{50}$ for the VR intervention group. This is the most commonly used questionnaire in VR research. ${ }^{32}$ Higher scores indicate higher side effects. The SSQ includes three subscales including nausea, oculomotor and disorientation. Good internal consistency of the SSQ has been reported $(\alpha=0.87) .{ }^{51}$

\section{Activity levels}

Actigraphy will be measured using a GENEActiv wristband. The GENEActiv is placed for 48 hours at baseline and is repeated for 48 hours at the end of intervention after follow-up session for all three groups. Variables from GENEActiv will be categorised into levels of sedentary, light and moderate activity. Decreased daytime motor activity has been found in individuals with apathy compared with those without apathy. ${ }^{52}$ It is expected that after the intervention, the VR group will spend more time pursuing non-sedentary activities than the active and passive control groups. It is also expected that both intervention groups will spend more time in non-sedentary behaviour after the intervention compared with baseline than the passive control group. All data will be processed using a customised script in RStudio. ${ }^{53}$

\section{Heart rate variability and galvanic skin response}

Physiological measures including heart rate variability and galvanic skin response will be measured using the Empatica E4 wrist-worn wireless device (Empatica, Boston, USA). The Empatica E4 will be fitted during intervention sessions 1 and 3 to analyse changes throughout the intervention for both the VR and active control groups. Heart rate variability will be derived from the interbeat interval recorded from the Empatica E4. Increased heart rate variability response has been associated with positive emotions. ${ }^{54}$ It is expected that a fully immersive experience provided to participants in the VR group will result in an increased heart rate variability response compared with the active control group during the reminiscence sessions.

Galvanic skin response will be measured through skin conductance to examine physiological responses during the reminiscence sessions. Exposure to autobiographical material has found to increase skin conductance response. ${ }^{40}$ It is expected that a fully immersive experience provided to participants in the VR group will result in higher physiological responses compared with the active control group during the reminiscence sessions. All data will be processed in MATLAB (MathWorks, Natick, MA, USA) using customised scripts.

\section{Speech}

Participants will be requested to perform four speech tasks including responding to a question and reading from a wordless picture book at the start and end of intervention sessions 1 and 3. For the first speech task, two different questions will be used and counterbalanced.
For example, 'describe your typical Sunday' and 'what did you do yesterday'. The response will be timed for $60 \mathrm{~s}$. For the second speech task, reading from a wordless picture book will be limited to a maximum time of $5 \mathrm{~min}$. A series of four books from the same author with similar content (a different book for each time) will be used and counterbalanced. All speech tasks will be recorded using a digital recorder with a lapel microphone attached to the participant at sternum level. Prosodic, formant, source and temporal aspects of speech will be examined. ${ }^{41}$ It is expected that there will be a higher activation of speech markers after the reminiscence sessions compared with before sessions and that activation of speech markers will be higher in the VR group compared with the active control group. Data will be analysed using Praat. ${ }^{55}$

\section{Staff questionnaire}

A staff questionnaire developed by the primary researcher will measure changes in aspects of the participants behaviour from the perspective of staff. This includes changes in social involvement, cognitive awareness, pain, activities of daily living, behaviour and communication. Questions are answered on a 5-point scale ranging from 'Not at all' to 'Very much so' and will assess improvement or deterioration during the period of the intervention.

\section{Session record}

The session record by Bender (see p291 ${ }^{56}$ will be completed for both the VR and active control group. Completed by researchers during the interventions process, this record will measure attendance to sessions, memory recall of reminiscence content, interaction, responsiveness and enjoyment on a 4-point scale. Participants will also be asked if they would like to do reminiscence again, and for the VR group, participants are asked if they prefer VR to a flat screen display.

\section{Apparatus}

VR software

For the VR group, off-the-shelf software will be used for reminiscence. This will include YouTube VR (developed by Google LLC) for the playback of videos. Wander (developed by Parkline Interactive) will be used to view places relevant to each participant, this application makes use of data from Google Street View. For the active control group, Google Street View and YouTube will be viewed on a laptop computer and the internet will also be used to source images from various websites.

\section{VR hardware}

The Oculus Quest ${ }^{57}$ HMD will be used to deliver the VR experience to participants. This is a standalone HMD with sensors built into the headset for tracking movement in the virtual environment.

\section{GENEActiv}

Activity will be measured using a GENEActiv wristworn accelerometer (Activinsights, Kimbolton, Cambridgeshire, UK). This is a lightweight, waterproof 
body-worn accelerometer that measures and tracks movement in all environments.

\section{Empatica E4}

Heart rate variability and galvanic skin response will be measured using Empatica E4 wristband (Empatica, Boston, USA). This is a medical-grade wristband that measures real-time physiological data.

\section{Sample size}

The required sample size was calculated using G*Power statistical analysis V.3.1.9.7, ${ }^{58}$ a prior sample size was calculated. The approximate sample size was calculated with an analysis of variance for repeated measures (large effect size, power $=0.80$ and $\alpha=0.05$ ). The large effect size was based on outcome of apathy using reminiscence therapy supported by internet based videos. ${ }^{23}$ The approximate sample size required was calculated to be $n=36$ (12 per group); therefore, to account for attrition, a total of 45 participants (15 per group) will be recruited.

\section{Recruitment}

A research nurse employed by the aged care provider has been assigned to recruit participants; in his/her absence, the primary investigator will undertake recruitment. Potentially suitable participants are identified by senior staff at the residential aged care facility in accordance with inclusion/exclusion criteria and a list is provided to the research nurse and primary investigator. Each group is allocated to a separate residential aged care site, this will assist with ensuring that adequate participant enrolment is achieved and that residents are blinded to the other groups. No honorarium will be offered for participation.

\section{Blinding and data collection}

The research team members conducting baseline and follow-up measures are blinded to group allocation. Research team members administering outcome measures will not have access to group allocation in the electronic database. Participants are blinded to the presence of the other groups assisted by allocating each group to a separate residential aged care site.

The reminiscence interview and intervention sessions are carried out by the primary researcher and an additional member of the research team who is not conducting baseline and follow-up measures.

\section{Statistical methods}

Data will be analysed to examine distributions and check for missing values and outliers. Descriptive statistics will be used to summarise the baseline characteristics. This is a per-protocol analysis, participants will be replaced if possible. The primary outcome at baseline and follow-up will be analysed using linear mixed modelling. Fixed factors will be group (VR, active control, passive control) and time (baseline, follow-up), with intercept of participant as a random factor. For comparisons between the two intervention groups with the usual care group, Helmert contrasts will be used where the contrasts will compare
(1) combined intervention groups with the usual care group and (2) the two interventions. Additional analysis will be performed including only participants that meet the criteria for a diagnosis of apathy using a cut-off score of 37.5. ${ }^{42}$ Further secondary analysis will be conducted to examine the influence of potential covariates including depression and cognition at baseline. Secondary and exploratory outcomes will be analysed as per the primary outcome.

\section{ETHICS AND DISSEMINATION \\ Informed consent}

Participants that are interested and meet the exclusion/ inclusion criteria are provided with written and verbal information about the study. A dedicated research nurse employed by the residential aged care facility or the primary researcher obtains informed consent (see online supplemental file 1). All participants are given the opportunity to discuss participation with family members or other responsible person close to the participant. Consent is continually monitored during the research by asking participants if they want to continue at the start and end of each session.

\section{Data management}

Each participant will be given a unique identification number that is stored in the electronic database. Research Electronic Data Capture, ${ }^{60}$ a secure web-based software platform, will be used for storing all data. All data are stored using anonymous codes. Codes with paper data are in lockable storage and can only be accessed by research staff involved in the project. Data will be stored for a minimum of 5 years from study completion.

\section{Harms}

Potential for harms is minimal. A risk associated with using HMDs is VR sickness characterised by side effects that are like those experienced in motion sickness from air, sea or land travel. If a participant does experience any side effects, they will be monitored by the researchers until symptoms subside. If symptoms persist longer than the research session, the participant will be referred to the nurse on duty and will be monitored by staff at the aged care facility until symptoms subside.

The process of reminiscence therapy has the potential to raise memories that are distressing. The use of reminiscence therapy will focus on memories that are positive to the participant, negative memories will be avoided. This will avoid causing undue stress to the participant due to memories that may be distressing. It is possible that even positive memories can cause emotional reactions. If any distress does occur, validation, reassurance and distraction approaches will be used depending on the response. ${ }^{38}$

If a participant scores 10 or higher on the GDS at baseline or follow-up, staff at the residential aged facility will 
be made aware and will follow-up and offer support, as necessary.

\section{Data monitoring}

Due to the relatively small scale of the trial, there will be no data monitoring committee, interim analyses and auditing trial conduct.

\section{Protocol amendments}

Any protocol amendments will be submitted to the University of South Australia Human Research Ethics Committee for approval. The primary investigator will update the trial registry after amendments have been approved.

\section{Access to data}

Study staff including the primary investigator and research assistants will have access to raw data files. Access to data for each study staff member is limited to functions they are responsible for. Investigators outside of the study may request access to datasets through the corresponding author.

\section{Dissemination}

The results from this study will be disseminated through manuscript publications and national/international conferences. All study investigators will be eligible for authorship depending on contributions to the manuscripts, the use of professional writers is not intended.

Contributors DS and TL: conception of the work. DS: drafted the manuscript. DS, HADK, MC and TL: revised the work critically for important intellectual content and have read and approved the manuscript.

Funding DS was supported by the Australian Government Research Training Program Scholarship. TL and HADK were funded by National Health and Medical Research Council Dementia Research Leadership Fellowships (GNT1136269 \& GNT1135676, respectively). The above listed funding bodies did not contribute to the design of the study, data collection, analysis, interpretation of data, or writing the manuscript.

Competing interests None declared.

Patient consent for publication Not required.

Provenance and peer review Not commissioned; externally peer reviewed.

Supplemental material This content has been supplied by the author(s). It has not been vetted by BMJ Publishing Group Limited (BMJ) and may not have been peer-reviewed. Any opinions or recommendations discussed are solely those of the author(s) and are not endorsed by BMJ. BMJ disclaims all liability and responsibility arising from any reliance placed on the content. Where the content includes any translated material, BMJ does not warrant the accuracy and reliability of the translations (including but not limited to local regulations, clinical guidelines, terminology, drug names and drug dosages), and is not responsible for any error and/or omissions arising from translation and adaptation or otherwise.

Open access This is an open access article distributed in accordance with the Creative Commons Attribution Non Commercial (CC BY-NC 4.0) license, which permits others to distribute, remix, adapt, build upon this work non-commercially, and license their derivative works on different terms, provided the original work is properly cited, appropriate credit is given, any changes made indicated, and the use is non-commercial. See: http://creativecommons.org/licenses/by-nc/4.0/.

\section{ORCID iD}

Dimitrios Saredakis http://orcid.org/0000-0002-3808-4479
REFERENCES

1 Nijsten JMH, Leontjevas R, Pat-El R, et al. Apathy: risk factor for mortality in nursing home patients. J Am Geriatr Soc 2017;65:2182-9.

2 Robert P, Lanctôt KL, Agüera-Ortiz L, et al. Is it time to revise the diagnostic criteria for apathy in brain disorders? the 2018 international consensus group. Eur Psychiatry 2018;54:71-6.

3 van Dalen JW, van Wanrooij LL, Moll van Charante EP, et al. Association of apathy with risk of incident dementia: a systematic review and meta-analysis. JAMA Psychiatry 2018;75:1012-21.

4 Ellis JM, Doyle CJ, Selvarajah S. The relationship between apathy and participation in therapeutic activities in nursing home residents with dementia: evidence for an association and directions for further research. Dementia 2016;15:494-509.

5 Boyle PA, Malloy PF, Salloway S, et al. Executive dysfunction and apathy predict functional impairment in Alzheimer disease. Am J Geriatr Psychiatry 2003;11:214-21.

6 Brodaty H, Draper B, Saab D, et al. Psychosis, depression and behavioural disturbances in Sydney nursing home residents: prevalence and predictors. Int J Geriatr Psychiatry 2001;16:504-12.

7 Rajkumar AP, Ballard C, Fossey J, et al. Apathy and its response to antipsychotic review and nonpharmacological interventions in people with dementia living in nursing homes: WHELD, a factorial cluster randomized controlled trial. J Am Med Dir Assoc 2016;17:741-7.

8 Majic T, Pluta J-P, Mell T, et al. The pharmacotherapy of neuropsychiatric symptoms of dementia: a cross-sectional study in 18 homes for the elderly in Berlin. Dtsch Arztebl Int 2010;107:320-7.

9 Wood S, Cummings JL, Hsu MA, et al. The use of the neuropsychiatric inventory in nursing home residents. Characterization and measurement. Am J Geriatr Psychiatry 2000;8:75-83.

10 Zuidema SU, Derksen E, Verhey FRJ, et al. Prevalence of neuropsychiatric symptoms in a large sample of Dutch nursing home patients with dementia. Int J Geriatr Psychiatry 2007;22:632-8.

11 Leone E, Deudon A, Bauchet M, et al. Management of apathy in nursing homes using a teaching program for care staff: the STIMEHPAD study. Int J Geriatr Psychiatry 2013;28:383-92.

12 Chase TN. Apathy in neuropsychiatric disease: diagnosis, pathophysiology, and treatment. Neurotox Res 2011;19:266-78.

13 Bogdan A, Manera V, Koenig A, et al. Pharmacologic approaches for the management of apathy in neurodegenerative disorders. Front Pharmacol 2019;10:1581-81.

14 Theleritis CG, Siarkos KT, Politis AM. Unmet needs in pharmacological treatment of apathy in Alzheimer's disease: a systematic review. Front Pharmacol 2019;10:1108-08.

15 Manera V, Abrahams S, Agüera-Ortiz L, et al. Recommendations for the nonpharmacological treatment of apathy in brain disorders. Am J Geriatr Psychiatry 2020;28:410-20.

16 Woods B, O'Philbin L, Farrell EM, et al. Reminiscence therapy for dementia. Cochrane Database Syst Rev 2018;192.

17 Macleod F, Storey L, Rushe T, et al. Towards an increased understanding of reminiscence therapy for people with dementia: a narrative analysis. Dementia 2020:147130122094127.

18 Riedl M, Mantovan F, Them C. Being a nursing home resident: a challenge to one's identity. Nurs Res Pract 2013;2013:1-9.

19 Bohlmeijer E, Roemer M, Cuijpers P, et al. The effects of reminiscence on psychological well-being in older adults: a metaanalysis. Aging Ment Health 2007;11:291-300.

20 Amieva H, Robert PH, Grandoulier A-S, et al. Group and individual cognitive therapies in Alzheimer's disease: the ETNA3 randomized trial. Int Psychogeriatr 2016;28:707-17.

21 Hsieh C-J, Chang C, Su S-F, et al. Reminiscence group therapy on depression and apathy in nursing home residents with mild-tomoderate dementia. J Exp Clin Med 2010;2:72-8.

22 Lazar A, Thompson H, Demiris G. A systematic review of the use of technology for reminiscence therapy. Health Educ Behav 2014;41:51S-61.

23 Manav Al, Simsek N. The effect of reminiscence therapy with internet-based videos on cognitive status and apathy of older people with mild dementia. J Geriatr Psychiatry Neurol 2019;32:104-13.

24 Maples-Keller JL, Yasinski C, Manjin N, et al. Virtual reality-enhanced extinction of phobias and post-traumatic stress. Neurotherapeutics 2017;14:554-63.

25 Tashjian VC, Mosadeghi S, Howard AR, et al. Virtual reality for management of pain in hospitalized patients: results of a controlled trial. JMIR Ment Health 2017;4:e9.

26 Maples-Keller JL, Bunnell BE, Kim S-J, et al. The use of virtual reality technology in the treatment of anxiety and other psychiatric disorders. Harv Rev Psychiatry 2017;25:103-13. 
27 Brimelow RE, Dawe B, Dissanayaka N. Preliminary research: virtual reality in residential aged care to reduce apathy and improve mood. Cyberpsychol Behav Soc Netw 2020;23:165-70.

28 Benoit M, Guerchouche R, Petit P-D, et al. Is it possible to use highly realistic virtual reality in the elderly? A feasibility study with imagebased rendering. Neuropsychiatr Dis Treat 2015;11:557-63.

29 Manera V, Chapoulie E, Bourgeois J, et al. A feasibility study with image-based rendered virtual reality in patients with mild cognitive impairment and dementia. PLoS One 2016;11:e0151487.

30 Saredakis D, Keage HA, Corlis M, et al. Using virtual reality to improve apathy in residential aged care: mixed methods study. $J$ Med Internet Res 2020;22:e17632.

31 Huygelier H, Schraepen B, van Ee R, et al. Acceptance of immersive head-mounted virtual reality in older adults. Sci Rep 2019;9:4519.

32 Rebenitsch L, Owen C. Review on cybersickness in applications and visual displays. Virtual Real 2016;20:101-25.

33 Saredakis D, Szpak A, Birckhead B, et al. Factors associated with virtual reality sickness in head-mounted displays: a systematic review and meta-analysis. Front Hum Neurosci 2020;14:96.

34 Dermody $\mathrm{G}$, Whitehead L, Wilson $\mathrm{G}$, et al. The role of virtual reality in improving health outcomes for community-dwelling older adults: systematic review. J Med Internet Res 2020;22:e17331.

35 Chen J-H, Chan D-CD, Kiely DK, et al. Terminal trajectories of functional decline in the long-term care setting. J Gerontol A Biol Sci Med Sci 2007;62:531-6.

36 Chan A-W, Tetzlaff JM, Gøtzsche PC, et al. SPIRIT 2013 explanation and elaboration: guidance for protocols of clinical trials. BMJ 2013;346:e7586.

37 Jorm AF, Mackinnon AJ, Henderson AS, et al. The psychogeriatric assessment scales: a multi-dimensional alternative to categorical diagnoses of dementia and depression in the elderly. Psychol Med 1995;25:447-60.

38 Westphal A, Callega D, LoGiudice D. Using reminiscence with people with dementia in sub-acute \& acute care, 2017. Available: http:// medicine.unimelb.edu.au/_data/assets/pdf_file/0020/2471312/ Using-reminiscence-with-people-with-dementia-in-acute-andsubacute-care-manual.pdf

39 Cummings J, Friedman JH, Garibaldi G, et al. Apathy in neurodegenerative diseases: recommendations on the design of clinical trials. J Geriatr Psychiatry Neurol 2015;28:159-73.

40 Treusch Y, Page J, van der Luijt C, et al. Emotional reaction in nursing home residents with dementia-associated apathy: a pilot study. Geriatric Mental Health Care 2015;3:1-6.

41 König A, Linz N, Zeghari R, et al. Detecting apathy in older adults with cognitive disorders using automatic speech analysis. $J$ Alzheimers Dis 2019;69:1183-93.

42 Marin RS, Biedrzycki RC, Firinciogullari S. Reliability and validity of the apathy evaluation scale. Psychiatry Res 1991;38:143-62.

43 Noone P. Addenbrooke's cognitive examination-III. Occup Med 2015;65:418-20.
44 Hsieh S, Schubert S, Hoon C, et al. Validation of the Addenbrooke's cognitive examination III in frontotemporal dementia and Alzheimer's disease. Dement Geriatr Cogn Disord 2013;36:242-50.

45 Kurlowicz L, Greenberg SA. The geriatric depression scale (GDS). AJN, American Journal of Nursing 2007;107:67-8.

46 D'Ath P, Katona P, Mullan E, et al. Screening, detection and management of depression in elderly primary care attenders. I: the acceptability and performance of the 15 item geriatric depression scale (GDS15) and the development of short versions. Fam Pract 1994;11:260-6.

47 Logsdon RG, Gibbons LE, McCurry SM, et al. Assessing quality of life in older adults with cognitive impairment. Psychosom Med 2002;64:510-9.

48 Thorgrimsen L, Selwood A, Spector A, et al. Whose quality of life is it anyway? the validity and reliability of the quality of LifeAlzheimer's disease (QoL-AD) scale. Alzheimer Dis Assoc Disord 2003;17:201-8.

49 Hughes ME, Waite LJ, Hawkley LC, et al. A short scale for measuring loneliness in large surveys: results from two population-based studies. Res Aging 2004;26:655-72.

50 Kennedy RS, Lane NE, Berbaum KS, et al. Simulator sickness questionnaire: an enhanced method for quantifying simulator sickness. Int J Aviat Psychol 1993;3:203-20.

51 Bouchard S, Robillard G, Renaud P. Revising the factor structure of the simulator sickness questionnaire. Ann Rev Cyber Telemed 2007;5:128-37.

52 David R, Mulin E, Friedman L, et al. Decreased daytime motor activity associated with apathy in Alzheimer disease: an actigraphic study. Am J Geriatr Psychiatry 2012;20:806-14.

53 RStudio: Integrated Development for R [program] 2020 https:// rstudio.com/

54 Kok BE, Fredrickson BL. Upward spirals of the heart: autonomic flexibility, as indexed by vagal tone, reciprocally and prospectively predicts positive emotions and social connectedness. Biol Psychol 2010;85:432-6.

55 Praat: doing phonetics by computer [program]. 6.1.16 version 2020 https://www.fon.hum.uva.nl/praat/

56 Gibson F. Reminiscence and life story work: a practice guide. 4th edn. Pennsylvania: Jessica Kingsley Publishers, 2012.

57 Oculus. Oculus quest 2019. Available: https://support.oculus.com/

58 Faul F, Erdfelder E, Lang A-G, et al. G*Power 3: a flexible statistical power analysis program for the social, behavioral, and biomedical sciences. Behav Res Methods 2007;39:175-91.

59 Clarke DE, Reekum Rvan, Simard M, et al. Apathy in dementia: an examination of the psychometric properties of the apathy evaluation scale. J Neuropsychiatry Clin Neurosci 2007;19:57-64.

60 Harris PA, Taylor R, Thielke R, et al. Research electronic data capture (REDCap)--a metadata-driven methodology and workflow process for providing translational research informatics support. J Biomed Inform 2009:42:377-81. 